Article

\title{
Association between Nafamostat Mesylate and In-Hospital Mortality in Patients with Coronavirus Disease 2019: A Multicenter Observational Study
}

\author{
Ryota Inokuchi ${ }^{1}\left(\mathbb{D}\right.$, Toshiki Kuno ${ }^{2}$, Jun Komiyama ${ }^{1,3}$, Kazuaki Uda ${ }^{4}$, Yoshihisa Miyamoto ${ }^{5}$, Yuta Taniguchi ${ }^{3,4}{ }^{(\mathbb{D}}$, \\ Toshikazu Abe 1,6 , Miho Ishimaru ${ }^{1}$, Motohiko Adomi 1,4 ${ }^{\mathbb{D}}$, Nanako Tamiya ${ }^{1,4}$ and Masao Iwagami ${ }^{1,4, *}$ \\ 1 Department of Health Services Research, Faculty of Medicine, University of Tsukuba, \\ Tsukuba 305-8575, Japan; inokuchir-icu@md.tsukuba.ac.jp (R.I.); jun.komi33@gmail.com (J.K.); \\ abetoshi111@gmail.com (T.A.); ishimaru.miho.kf@u.tsukuba.ac.jp (M.I.); \\ adomimotohiko@gmail.com (M.A.); ntamiya@md.tsukuba.ac.jp (N.T.) \\ 2 Montefiore Medical Center, Division of Cardiology, Albert Einstein College of Medicine, \\ New York, NY 10461, USA; tkuno@montefiore.org \\ 3 Graduate School of Comprehensive Human Sciences, University of Tsukuba, Tsukuba 305-8575, Japan; \\ taniguchi.yuta.ma@alumni.tsukuba.ac.jp \\ 4 Health Services Research and Development Center, University of Tsukuba, Tsukuba 305-8575, Japan; \\ udakazuaki-tky@umin.ac.jp \\ 5 National Cancer Center, Institute for Cancer Control, Tokyo 104-0045, Japan; ymiyamoto70@gmail.com \\ 6 Department of Emergency and Critical Care Medicine, Tsukuba Memorial Hospital, Tsukuba 305-8575, Japan \\ * Correspondence: iwagami-tky@umin.ac.jp; Tel.: +81-(0)29-853-8849; Fax: +81-(0)29-853-8849
}

check for updates

Citation: Inokuchi, R.; Kuno, T.; Komiyama, J.; Uda, K.; Miyamoto, Y.; Taniguchi, Y.; Abe, T.; Ishimaru, M.; Adomi, M.; Tamiya, N.; et al. Association between Nafamostat Mesylate and In-Hospital Mortality in Patients with Coronavirus Disease 2019: A Multicenter Observational Study. J. Clin. Med. 2022, 11, 116. https://doi.org/10.3390/ jcm11010116

Academic Editor: Emanuele Nicastri

Received: 6 November 2021 Accepted: 23 December 2021 Published: 26 December 2021

Publisher's Note: MDPI stays neutral with regard to jurisdictional claims in published maps and institutional affiliations.

Copyright: (C) 2021 by the authors. Licensee MDPI, Basel, Switzerland. This article is an open access article distributed under the terms and conditions of the Creative Commons Attribution (CC BY) license (https:// creativecommons.org/licenses/by/ $4.0 /)$.

\begin{abstract}
Nafamostat mesylate may be effective against coronavirus disease 2019 (COVID-19). However, it is not known whether its use is associated with reduced in-hospital mortality in clinical practice. We conducted a retrospective observational study to evaluate the effect of nafamostat mesylate in patients with COVID-19 using the Medical Data Vision Co. Ltd. hospital-based database in Japan. We compared patients with COVID-19 who were $(n=121)$ and were not $(n=15,738)$ administered nafamostat mesylate within 2 days of admission between January and December 2020. We conducted a 1:4 propensity score matching with multiple imputations for smoking status and body mass index and combined the 20 imputed propensity score-matched datasets to obtain the adjusted odds ratio for in-hospital mortality. Crude in-hospital mortality was $13.2 \%(16 / 121)$ and $5.0 \%(790 / 15,738)$, respectively. In the propensity score-matched analysis with multiple imputations, the adjusted odds ratio (use vs. no use of nafamostat mesylate) for in-hospital mortality was 1.27 (95\% confidence interval: $0.61-2.64 ; p=0.52$ ). Sensitivity analyses showed similar results. The results of this retrospective observational study did not support an association between nafamostat mesylate and improved in-hospital outcomes in patients with COVID-19, although further studies with larger sample sizes are warranted to assess the generalizability of our findings.
\end{abstract}

Keywords: coronavirus disease 2019; in-hospital mortality; nafamostat mesylate

\section{Introduction}

The coronavirus disease 2019 (COVID-19) pandemic is caused by severe acute respiratory syndrome coronavirus 2 (SARS-CoV-2) infection. Although worldwide vaccination against SARS-CoV-2 is underway [1], it is imperative that researchers quickly identify suitable drugs for repurposing as COVID-19-specific therapies.

Nafamostat mesylate (NM) is a serine proteinase inhibitor that has been used in Japan for over 30 years to treat disseminated intravascular coagulation and pancreatitis [2]. Experimental studies have shown that SARS-CoV-2 exerts an effect on human angiotensinconverting enzyme 2, enabling it to invade cells and establish infection [3]. In 293FT cells (derived from human fetal kidneys) that ectopically express angiotensin-converting enzyme 
2, NM prevents SARS-CoV-2 spike protein-initiated fusion by inhibiting protease [4]. Thus, it can be hypothesized that NM is effective against COVID-19.

Recent previous basic experimental [5] and case reports or series [2,6-8] have suggested the potential effectiveness of NM against COVID-19. However, to our knowledge, neither clinical trials nor observational studies, except for a recent small phase 2 open-label, randomized controlled trial (RCT) [9] have demonstrated an association between NM and reduced mortality. Therefore, we aimed to evaluate the effect of NM in patients with COVID-19 using a large-scale in-patient database in Japan.

\section{Materials and Methods}

\subsection{Data Source}

We conducted a retrospective observational cohort study using the Medical Data Vision (MDV) Co., Ltd. (Tokyo, Japan) hospital-based database. The MDV is a private database that has been used to compile data on healthcare resource consumption at participating hospitals since 2008, based on information from the Japanese Diagnosis Procedure Combination (DPC) fixed-payment reimbursement system. The MDV database currently covers over 350 facilities in Japan, accounting for more than $20 \%$ of acute-care hospitals that use the DPC, and includes data on 32 million patients of all ages. The MDV database contains the following information: age; sex; height; body weight; admission and discharge dates; discharge status; level of consciousness; comorbidities on admission; smoking status; primary admission diagnosis according to the International Classification of Diseases, Tenth Revision (ICD-10) codes; and daily records of drugs, blood products, and procedures (including noninvasive positive-pressure ventilation [NPPV], mechanical ventilation, renal replacement therapy [RRT], and extracorporeal membrane oxygenation [ECMO]). A previous validation study of DPC data [10] suggested high sensitivity and specificity for procedural records, but high or moderate sensitivity for most diagnoses.

\subsection{Study Participants and Exposure Variable}

We included patients $\geq 18$ years of age who met the following criteria: (1) admission diagnosis of COVID-19 in accordance with ICD-10 code U071 and (2) discharged between 1 January 2020 and 31 December 2020. We excluded patients discharged on the day of admission to avoid immortal time bias [11], meaning that patients needed to survive to receive NM. Additionally, only initial hospitalizations were included for each patient; readmissions were excluded from the study. We compared the outcomes of patients who were administered $\mathrm{NM}$ within 2 days after admission (NM group) to the outcomes of those who were not administered NM (control group).

\subsection{Outcome and Covariates}

The primary outcome was in-hospital mortality. Covariates included age; sex; prior diagnoses of diabetes mellitus, cardiac disease, cerebral infarction, liver disease, chronic lung disease, cancer, and chronic kidney disease; Charlson comorbidity index [12]; body mass index (BMI); smoking status; level of consciousness at the time of admission; use of antiplatelet or anticoagulant drugs (including vitamin $\mathrm{K}$ antagonists and direct oral anticoagulants) at the time of admission; transfer from another hospital; and admission to the intensive care unit (ICU) (ICD-10 codes are shown in Table S1). For the purpose of analysis, we categorized levels of consciousness, according to the Japan Coma Scale (JCS), as follows: alert, JCS 0; awake without stimulation, JCS 1-3; arousable with stimulation, JCS 10-30; and unarousable, JCS 100-300. The JCS is widely used in Japan and correlates well with the Glasgow Coma Scale [13]. In addition, we identified patients who received the following treatments within 2 days of admission: NPPV, mechanical ventilation, RRT, ECMO, transfusion (including red blood cell concentrates, fresh frozen plasma, and platelet concentrates), vasoactive agents (including norepinephrine, dobutamine, and vasopressin), intravenous antibiotics, anticoagulants (including heparin and daluteparin), and steroids (including dexamethasone and other oral and intravenous steroids). 


\subsection{Statistical Analysis}

Baseline characteristics that were measured as continuous or categorical variables were summarized. Categorical data are expressed as percentages. Normally and non-normally distributed variables are expressed as mean (standard deviation) and median (interquartile range), respectively. The chi-square test was used to compare categorical data, except when the expected cell counts were five or fewer, in which case Fisher's exact test was used. Continuous variables were compared using Welch's t-test or the Mann-Whitney U test, depending on the distribution of the data.

For our study population, we grouped patients based on the presence or absence of each diagnostic or procedural code, assuming that patients without a code did not have the corresponding condition. Therefore, no covariate data were missing, except for smoking status and BMI. There were missing values for BMI and smoking status on admission, which may have affected the results. Therefore, before propensity score matching, we replaced each missing value with a set of substituted plausible values using a multistep approach. First, we performed multiple imputations to account for missing data on smoking status and BMI [14]. We replaced each missing value with a set of substituted plausible values by creating 20 filled-in complete datasets, with 10 iterations per dataset, using the multiple imputations by chained equations method [15]. The following covariates were used in the imputation model: age, sex, Charlson comorbidity index, level of consciousness, use of antiplatelet or anticoagulant drugs, ICU admission, transfer from another hospital, comorbidities, treatments performed within 2 days of admission, and in-hospital mortality. Second, to estimate the propensity score, we fitted a logistic regression model for NM use as a function of the patient and hospital factors (i.e., age, sex, comorbidities, BMI, smoking status, level of consciousness, use of antiplatelet or anticoagulant drugs, ICU admission, transfer from another hospital, and the number of beds) and treatments performed within 2 days of admission (i.e., NPPV, mechanical ventilation, RRT, ECMO, transfusion [including red blood cell concentrates, fresh frozen plasma, and platelet concentrates], vasoactive agents [including norepinephrine, dobutamine, and vasopressin], intravenous antibiotics, and anticoagulants [including unfractionated or low-molecular-weight heparin, dexamethasone, and other steroids]). We selected these covariates based on clinical importance to calculate the propensity score [16]. Third, propensity score matching was performed for each imputed dataset using 1:4 nearest-neighbor matching based on the estimated propensity score of each patient. A match occurred when a patient in the NM-user group had an estimated propensity score within 0.2 standard deviations of that of a patient in the non-user group [17,18]. Balance was determined using standardized mean differences. To assess balance after matching, we calculated the standardized mean difference for each dataset and considered values $<0.1$ to be acceptable [19]. Fourth, effect estimates were determined and the results were pooled using Rubin's rules [20]. Effect estimates are presented as odds ratios (ORs) for binary outcome data with corresponding $95 \%$ confidence intervals. Imputed and matched data are presented as pooled data.

A two-sided $p<0.05$ was considered statistically significant. We conducted sensitivity analyses to test the robustness of our findings. First, we excluded patients who had undergone intermittent or continuous RRT from the study population, as NM is sometimes used for this purpose in Japan [21]. Second, we analyzed the outcomes using complete cases for BMI and smoking status.

Data were analyzed using JMP 15.1 (SAS Institute Inc., Cary, NC, USA), Stata MP15.1 (STATA Corp., College Station, TX, USA), and R version 4.1.1 (R Foundation for Statistical Computing, Vienna, Austria) with the mice, MatchThem, and cobalt survey packages.

\section{Results}

We included 15,859 patients after applying the inclusion and exclusion criteria (Figure 1). Of these, $2378(15.1 \%)$ and $2784(17.6 \%)$ patients from the NM and non-NM groups had missing data on BMI and smoking status, respectively. 


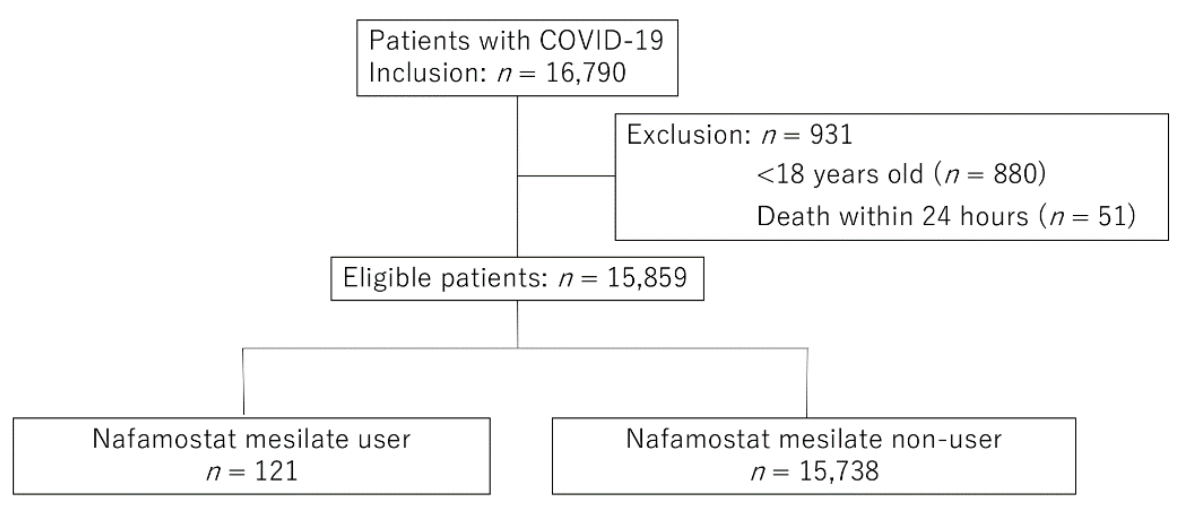

Figure 1. Flow chart of the patient selection process. COVID-19, coronavirus disease 2019.

Tables 1 and 2 show the baseline characteristics of the unmatched groups after multiple imputations. The unmatched data indicated that patients administered NM were more likely to be older males; have the comorbidities of diabetes mellitus and chronic kidney disease; have a high BMI; be in a comatose state; use antiplatelet drugs; have been transferred from another hospital; be admitted to a large-scale hospital or the ICU; be administered antibiotics, heparin, vasopressors, steroids, or transfusions; and require mechanical ventilation, RRT, or ECMO within 2 days of admission. Propensity score matching produced balanced, well-matched treatment groups for each set of imputed and pooled data (Figure 2 and Table S2).

Table 1. Unmatched patient characteristics categorized according to nafamostat mesylate use.

\begin{tabular}{|c|c|c|}
\hline & \multicolumn{2}{|c|}{ Unmatched Group } \\
\hline & Nafamostat Mesylate & Control \\
\hline Number of patients & 121 & 15,738 \\
\hline Age (years), mean $\pm \mathrm{SD}^{1}$ & $69.9 \pm 15.0$ & $61.8 \pm 22.2$ \\
\hline Male $(\%)$ & 68.6 & 57.1 \\
\hline \multicolumn{3}{|l|}{ Charlson comorbidity index (\%) } \\
\hline 0 & 43.8 & 52.1 \\
\hline 1 & 9.9 & 9.1 \\
\hline 2 & 25.6 & 16.4 \\
\hline 3 & 8.3 & 5.8 \\
\hline$\geq 4$ & 12.4 & 16.6 \\
\hline Diabetes mellitus (\%) & 40.5 & 22.5 \\
\hline Ischemic heart disease (\%) & 5.8 & 7.7 \\
\hline Cirrhosis $(\%)$ & 0.8 & 1.4 \\
\hline Chronic lung disease (\%) & 18.2 & 17.9 \\
\hline Cancer $(\%)$ & 12.4 & 13.5 \\
\hline Chronic kidney disease (\%) & 24.8 & 6.4 \\
\hline \multicolumn{3}{|l|}{ Body mass index (\%) } \\
\hline$<18.5$ & 10.4 & 16.3 \\
\hline $18.5-25.0$ & 59.1 & 58.0 \\
\hline $25-30$ & 20.0 & 19.1 \\
\hline$\geq 30$ & 10.4 & 6.6 \\
\hline Smoking $(\%)$ & 35.9 & 34.1 \\
\hline \multicolumn{3}{|l|}{ Japan Coma Scale (\%) } \\
\hline 0 (clear) & 66.1 & 83.9 \\
\hline 1-3 (delirium) & 22.3 & 12.7 \\
\hline 10-30 (somnolence) & 4.1 & 2.3 \\
\hline 100-300 (coma) & 7.4 & 1.1 \\
\hline $\mathrm{VKA}^{2}(\%)$ & 1.7 & 1.5 \\
\hline $\operatorname{DOAC}^{3}(\%)$ & 4.1 & 4.3 \\
\hline Antiplatelet $(\%)$ & 9.1 & 6.1 \\
\hline Interhospital transfer (\%) & 16.5 & 5.9 \\
\hline
\end{tabular}


Table 1. Cont.

\begin{tabular}{ccc}
\hline & \multicolumn{2}{c}{ Unmatched Group } \\
\cline { 2 - 3 } & Nafamostat Mesylate & Control \\
\hline Number of beds $(\%)$ & 2.5 & 6.5 \\
$<200$ & 33.9 & 56.6 \\
$200-400$ & 63.6 & 36.9 \\
$\geq 400$ & 37.2 & 4.6 \\
\hline ICU & admission (\%) & \\
\hline
\end{tabular}

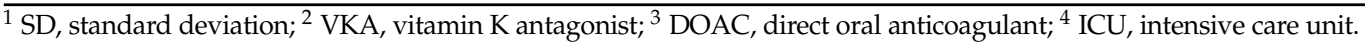

Table 2. Treatment within 2 days of admission in the unmatched cohort.

\begin{tabular}{ccc}
\hline & Nafamostat Mesylate & Control \\
\hline Initial antibiotics (\%) & 25.6 & 7.8 \\
Heparin (\%) & 14.0 & 2.6 \\
Daluteparin (\%) & 0.8 & 0.1 \\
Dobutamine (\%) & 2.5 & 0.2 \\
Noradrenalin (\%) & 14.9 & 1.0 \\
Vasopressin (\%) & 4.1 & 0.1 \\
Steroids & & \\
Dexamethasone (\%) & 19.0 & 4.9 \\
Other steroids (\%) & 19.8 & 6.1 \\
Blood transfusion (\%) & & \\
Red blood cells (\%) & 4.1 & 0.9 \\
Platelets (\%) & 1.7 & 0.1 \\
Fresh frozen plasma (\%) & 2.5 & 0.0 \\
Oxygen therapy (\%) & 47.9 & 26.3 \\
NPPV $^{1}(\%)$ & 1.7 & 0.3 \\
Mechanical ventilation (\%) & 19.8 & 1.7 \\
IRRT $^{2}(\%)$ & 17.4 & 1.3 \\
CRRT $^{3}$ (\%) & 10.7 & 0.1 \\
ECMO $^{4}(\%)$ & 2.5 & 0.1 \\
\hline
\end{tabular}

${ }^{1} \mathrm{NPPV}$, noninvasive positive-pressure ventilation; ${ }^{2}$ IRRT, intermittent renal replacement therapy; ${ }^{3}$ CRRT, continuous renal replacement therapy; ${ }^{4} \mathrm{ECMO}$, extracorporeal membrane oxygenation.

In the unmatched cohort, in-hospital mortality was 5.1\% $(806 / 15,859)$, and that of patients with or without NM was $13.2 \%(16 / 121)$ and $5.0 \%(790 / 15,738)$, respectively $(p<0.001)$. After propensity score matching, in-hospital mortality did not differ significantly between patients with or without NM (odds ratio, 1.27; $95 \%$ confidence interval: $0.61-2.64 ; p=0.52$ ) (Table 3 ). The results of sensitivity analyses (1) excluding patients who had undergone intermittent or continuous RRT and (2) using complete cases for BMI and smoking status, were similar to those of our main analysis, which did not suggest that NM had a statistically significant survival benefit among patients with COVID-19 (Table 3).

Table 3. Study outcomes after propensity score matching.

\begin{tabular}{lcc}
\hline & Effect Estimate & $p$-Value \\
\hline In-hospital mortality & & \\
$\quad$ No nafamostat mesylate & 1 (Reference) & 0.52 \\
$\quad$ Nafamostat mesylate & $1.27(0.61-2.64)$ & \\
$\begin{array}{l}\text { Sensitivity analyses (in-hospital mortality) } \\
\quad \text { No nafamostat mesylate }\end{array}$ & 1 (Reference) & \\
$\quad$ Nafamostat mesylate & & 0.94 \\
$\quad$ Exclusion of patients undergoing IRRT ${ }^{1}$ or & $1.03(0.39-2.71)$ & 0.46 \\
CRRT $^{2}$ & $1.32(0.62-2.82)$ & \\
$\quad$ Complete cases &
\end{tabular}

${ }^{1}$ IRRT, intermittent renal replacement therapy; ${ }^{2}$ CRRT, continuous renal replacement therapy. 


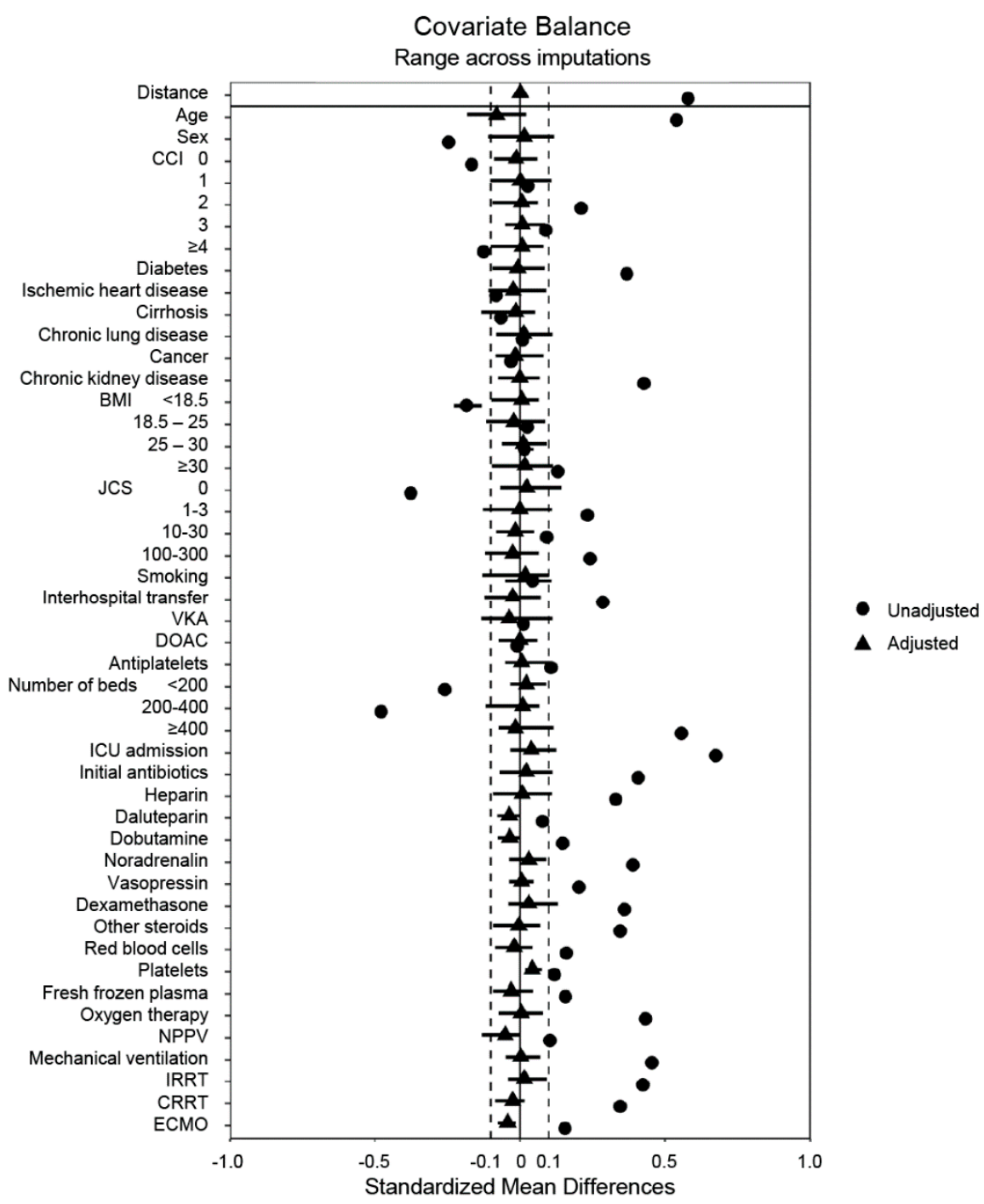

Figure 2. A summary plot of covariate balance before and after matching. BMI, body mass index; CCI, Charlson comorbidity index; CRRT, continuous renal replacement therapy; DOAC, direct oral anticoagulant; ECMO, extracorporeal membrane oxygenation; ICU, intensive care unit; IRRT, intermittent renal replacement therapy; JCS, Japan Coma Scale; NPPV, noninvasive positive-pressure ventilation; VKA, vitamin $\mathrm{K}$ antagonist.

\section{Discussion}

In the present observational study, which used a large-scale in-patient database, we compared in-hospital mortality between patients with COVID-19 who had and had not been administered NM. The results of propensity score matching after multiple imputations indicated no statistically significant differences in the outcomes of the two groups. Several subsequent sensitivity analyses also yielded the same conclusion.

Drug repurposing, that is, the use of existing commercially available drugs as an alternative treatment for a novel disease, is often attempted, and is a measure that greatly reduces the cost of drug development $[22,23]$. The adverse effects of repurposed drugs have often been studied previously. However, the current World Health Organization guidelines on drugs for COVID-19 only strongly recommend two drugs (corticosteroids and interleukin-6 receptor blockers) as a treatment for patients with severe or critical COVID19 [24]. Thus, verifying that existing commercial drugs are effective against COVID-19 by using a large-scale in-patient database is crucial.

We initially hypothesized that NM may improve patient outcomes, as experimental studies recently reported that NM inhibits the entry of SARS-CoV-2 into human epithelial 
cells [5,25], and several case reports have demonstrated the potential clinical benefits of NM [2,6-8]; however, we found no statistically significant association between NM and improvements in in-hospital mortality.

Our crude overall in-hospital mortality was $5.1 \%$, which is lower than a large study previously conducted in Japan (in-hospital mortality rate, 11.6\%), and the results showed lower mortality compared to other countries [26]. The reason may be few patients with severe COVID-19 were included (e.g., those admitted to the ICU and those requiring RRT or ECMO) in this study. We adjusted the severity by using propensity score matching; however, low in-hospital mortality and severity might affect our results.

Recently, a phase 2 open-label RCT in patients requiring nasal high-flow oxygen therapy and/or non-invasive mechanical ventilation with COVID-19 showed NM did not shorten the time to clinical improvement [9]. The result of the study supports our findings but further validation may be needed by larger RCTs.

This study has several limitations. First, it was a nonrandomized observational study. Therefore, the database did not include detailed clinical information on factors, such as symptoms, vital signs, and laboratory data. However, we included data from previous studies, which have associated advanced age, male sex, obesity, smoking, cardiovascular disease, diabetes mellitus, chronic lung disease, and cancer with increased COVID-19 mortality $[27,28]$, and considered those factors in our study. In addition, key factors forming part of the Acute Physiologic and Chronic Health Evaluation scoring system and severity of pneumonia (e.g., A-DROP score) that are widely used for predicting mortality or adjusting severity were assessed in our study (such as the use of vasopressors instead of mean arterial pressure, mechanical ventilation instead of oxygenation, RRT instead of renal function, and JCS instead of mental status). Second, we could not obtain data on newly approved COVID-19 drugs, such as remdesivir and ciclesonide, as they had not yet been assigned drug codes. Third, we were unable to adjust for the number of patients with COVID-19 seen at each facility during the study period, which may have affected the outcomes. Lastly, we were unable to assess long-term outcomes post-hospital discharge.

\section{Conclusions}

Despite using the current largest available sample size that we know of to date, we found no statistically significant association between NM administration and improvements in the incidence of in-hospital mortality in patients with COVID-19. Thus, administering NM to improve the aforementioned outcomes may not yet be justified. Further studies elucidating the benefits and disadvantages of administering NM to patients with COVID-19 are warranted.

Supplementary Materials: The following are available online at https:/ /www.mdpi.com/article/10 .3390/jcm11010116/s1, Table S1: The International Classification of Diseases, Tenth Revision (ICD-10) codes. Table S2: Summary of matched imputed datasets for in-hospital mortality (median).

Author Contributions: Conceptualization, R.I. and M.I. (Masao Iwagami); Data curation, T.K. and M.I. (Masao Iwagami); Formal analysis, R.I., J.K., K.U. and M.I. (Masao Iwagami); Writing—original draft preparation, R.I.; Writing—review and editing, T.K., J.K., K.U., Y.M., Y.T., T.A., M.I. (Miho Ishimaru), M.A., N.T. and M.I. (Masao Iwagami). All authors have read and agreed to the published version of the manuscript.

Funding: This research was funded by a Grant-in-Aid from the Ministry of Health, Labor, and Welfare in Japan, grant number 21AA2007.

Institutional Review Board Statement: The study was conducted according to the guidelines of the Declaration of Helsinki, and approved by the Institutional Review Board of the University of Tsukuba (protocol code \#1624-1 and 12 February 2021).

Informed Consent Statement: Patient consent was waived due to the observational nature of the study.

Data Availability Statement: The data presented in this study are available on request from the corresponding author. 
Acknowledgments: We would like to thank the employees of Medical Data Vision Co. Ltd., especially Masaki Nakamura and Shogo Atsuzawa, for their contributions to the preparation and interpretation of the claims data.

Conflicts of Interest: The authors declare no conflict of interest.

\section{References}

1. Sallam, M. COVID-19 Vaccine Hesitancy Worldwide: A Concise Systematic Review of Vaccine Acceptance Rates. Vaccines 2021, 9, 160. [CrossRef] [PubMed]

2. Takahashi, W.; Yoneda, T.; Koba, H.; Ueda, T.; Tsuji, N.; Ogawa, H.; Asakura, H. Potential mechanisms of nafamostat therapy for severe COVID-19 pneumonia with disseminated intravascular coagulation. Int. J. Infect. Dis. 2020, 102, 529-531. [CrossRef] [PubMed]

3. Zhang, H.; Penninger, J.M.; Li, Y.; Zhong, N.; Slutsky, A.S. Angiotensin-converting enzyme 2 (ACE2) as a SARS-CoV-2 receptor: Molecular mechanisms and potential therapeutic target. Intensive Care Med. 2020, 46, 586-590. [CrossRef]

4. Cho, T.; Han, H.-S.; Jeong, J.; Park, E.-M.; Shim, K.-S. A Novel Computational Approach for the Discovery of Drug Delivery System Candidates for COVID-19. Int. J. Mol. Sci. 2021, 22, 2815. [CrossRef]

5. Yamamoto, M.; Kiso, M.; Sakai-Tagawa, Y.; Iwatsuki-Horimoto, K.; Imai, M.; Takeda, M.; Kinoshita, N.; Ohmagari, N.; Gohda, J.; Semba, K.; et al. The Anticoagulant Nafamostat Potently Inhibits SARS-CoV-2 S Protein-Mediated Fusion in a Cell Fusion Assay System and Viral Infection In Vitro in a Cell-Type-Dependent Manner. Viruses 2020, 12, 629. [CrossRef] [PubMed]

6. Iwasaka, S.; Shono, Y.; Tokuda, K.; Nakashima, K.; Yamamoto, Y.; Maki, J.; Nagasaki, Y.; Shimono, N.; Akahoshi, T.; Taguchi, T. Clinical improvement in a patient with severe coronavirus disease 2019 after administration of hydroxychloroquine and continuous hemodiafiltlation with nafamostat mesylate. J. Infect. Chemother. 2020, 26, 1319-1323. [CrossRef] [PubMed]

7. Doi, K.; the COVID-UTH Study Group; Ikeda, M.; Hayase, N.; Moriya, K.; Morimura, N. Nafamostat mesylate treatment in combination with favipiravir for patients critically ill with COVID-19: A case series. Crit. Care 2020, 24, 1-4. [CrossRef]

8. Jang, S.; Rhee, J.-Y. Three cases of treatment with nafamostat in elderly patients with COVID-19 pneumonia who need oxygen therapy. Int. J. Infect. Dis. 2020, 96, 500-502. [CrossRef] [PubMed]

9. Zhuravel, S.V.; Khmelnitskiy, O.K.; Burlaka, O.O.; Gritsan, A.I.; Goloshchekin, B.M.; Kim, S.; Hong, K.Y. Nafamostat in hospitalized patients with moderate to severe COVID-19 pneumonia: A randomised Phase II clinical trial. EClinicalMedicine 2021, 41, 101169. [CrossRef]

10. Yamana, H.; Moriwaki, M.; Horiguchi, H.; Kodan, M.; Fushimi, K.; Yasunaga, H. Validity of diagnoses, procedures, and laboratory data in Japanese administrative data. J. Epidemiology 2017, 27, 476-482. [CrossRef]

11. Suissa, S. Immortal time bias in pharmaco-epidemiology. Am. J. Epidemiol. 2008, 167, 492-499. [CrossRef]

12. Quan, H.; Li, B.; Couris, C.M.; Fushimi, K.; Graham, P.; Hider, P.; Januel, J.-M.; Sundararajan, V. Updating and Validating the Charlson Comorbidity Index and Score for Risk Adjustment in Hospital Discharge Abstracts Using Data From 6 Countries. Am. J. Epidemiol. 2011, 173, 676-682. [CrossRef] [PubMed]

13. Ono, K.; Wada, K.; Takahara, T.; Shirotani, T. Indications for Computed Tomography in Patients With Mild Head Injury. Neurol. Med.-Chir. 2007, 47, 291-298. [CrossRef] [PubMed]

14. Rubin, D.B.; Schenker, N. Multiple imputation in health-care databases: An overview and some applications. Stat. Med. 1991, 10, 585-598. [CrossRef] [PubMed]

15. Aloisio, K.M.; Micali, N.; Swanson, S.A.; Field, A.; Horton, N.J. Analysis of Partially Observed Clustered Data using Generalized Estimating Equations and Multiple Imputation. Stata Journal: Promot. Commun. Stat. Stata 2014, 14, 863-883. [CrossRef]

16. Brookhart, M.A.; Schneeweiss, S.; Rothman, K.; Glynn, R.J.; Avorn, J.; Stürmer, T. Variable Selection for Propensity Score Models. Am. J. Epidemiol. 2006, 163, 1149-1156. [CrossRef]

17. Rosenbaum, P.R.; Rubin, D.B. Constructing a Control Group Using Multivariate Matched Sampling Methods That Incorporate the Propensity Score. Am. Stat. 1985, 39, 33-38.

18. Austin, P.C. An Introduction to Propensity Score Methods for Reducing the Effects of Confounding in Observational Studies. Multivar. Behav. Res. 2011, 46, 399-424. [CrossRef] [PubMed]

19. Austin, P.C. Balance diagnostics for comparing the distribution of baseline covariates between treatment groups in propensityscore matched samples. Stat. Med. 2009, 28, 3083-3107. [CrossRef]

20. Rubin, D.B. Propensity score methods. Am. J. Ophthalmol. 2010, 149, 7-9. [CrossRef]

21. Doi, K.; Nishida, O.; Shigematsu, T.; Sadahiro, T.; Itami, N.; Iseki, K.; Yuzawa, Y.; Okada, H.; Koya, D.; Kiyomoto, H.; et al. The Japanese Clinical Practice Guideline for acute kidney injury 2016. J. Intensiv. Care 2018, 6, 48. [CrossRef]

22. Attah, A.F.; Fagbemi, A.A.; Olubiyi, O.; Dada-Adegbola, H.; Oluwadotun, A.; Elujoba, A.; Babalola, C.P. Therapeutic Potentials of Antiviral Plants Used in Traditional African Medicine With COVID-19 in Focus: A Nigerian Perspective. Front. Pharmacol. 2021, 12, 596855. [CrossRef]

23. Abduljabbar, M.H. Clinical efficacy of Nafamostat Mesylate in combination with Favipiravir for COVID-19 pneumonia treatment review article. Ann. Med. Surg. 2021, 68, 102560. [CrossRef] [PubMed]

24. WHO. Update to living WHO guideline on drugs for COVID-19. BMJ 2021, 374, n1703. Available online: https://www.bmj.com/ content/374/bmj.n1703.long (accessed on 6 November 2021). 
25. Hoffmann, M.; Schroeder, S.; Kleine-Weber, H.; Müller, M.A.; Drosten, C.; Pöhlmann, S. Nafamostat Mesylate Blocks Activation of SARS-CoV-2: New Treatment Option for COVID-19. Antimicrob. Agents Chemother. 2020, 64, e00754-20. [CrossRef]

26. Matsunaga, N.; Hayakawa, K.; Terada, M.; Ohtsu, H.; Asai, Y.; Tsuzuki, S.; Suzuki, S.; Toyoda, A.; Suzuki, K.; Endo, M.; et al. Clinical epidemiology of hospitalized patients with COVID-19 in Japan: Report of the COVID-19 REGISTRY JAPAN. Clin. Infect. Dis. 2020, 73, e3677-e3689. [CrossRef]

27. Jordan, R.E.; Adab, P.; Cheng, K.K. COVID-19: Risk factors for severe disease and death. BMJ 2020, 368, m1198. [CrossRef] [PubMed]

28. Kim, L.; Garg, S.; O’Halloran, A.; Whitaker, M.; Pham, H.; Anderson, E.J.; Armistead, I.; Bennett, N.M.; Billing, L.; Como-Sabetti, K.; et al. Risk Factors for Intensive Care Unit Admission and In-hospital Mortality Among Hospitalized Adults Identified through the US Coronavirus Disease 2019 (COVID-19)-Associated Hospitalization Surveillance Network (COVID-NET). Clin. Infect. Dis. 2020, 72, e206-e214. [CrossRef] [PubMed] 